\title{
Segmentation of Crescent Sand Dunes in High Resolution Satellite Images using a Support Vector Machine for Allometry
}

\author{
M. A. Azzaoui ${ }^{1}$, L. Masmoudi ${ }^{2}$ \\ Laboratoire Conception et Systèmes \\ Électronique, signaux et \\ Informatique Université Mohammed \\ V de Rabat, Morocco
}

\author{
H. El Belrhiti ${ }^{3}$ \\ Département des Sciences \\ Fondamentales et Appliquées \\ Institut Agronomique et Vétérinaire \\ Hassan II Rabat, Morocco
}

\author{
I. E. Chaouki ${ }^{4}$ \\ Département Informatique \\ Ecole Nationale des Sciences \\ Appliquées d'Agadir \\ Agadir, Morocco
}

\begin{abstract}
The study of sand dunes movement is essential to understand and prevent the desertification phenomenon, and collecting data from the field is a labor intensive task, as deserts contain usually a large number of sand dunes. We propose to use computer vision and machine learning algorithms, combined with remote sensing and specifically high resolution satellite images for collecting data about the position and characteristics of moving sand dunes. We focused on the fastest moving sand dunes called barchans, which are threatening the settlements in the region of Laayoune, Morocco. We developed a process with three stages: In the first stage, we used an image processing approach with cascading Haar features for the detection of dunes location. In the second stage, we used a support vector machine for the segmentation of contours, and in the third stage we used an algorithm to measure the allometric features of barchans dunes. We explored the collected data, and found relevant correlations between dunes length, and width, and horns sizes, which could be used as key indicators for dunes growth and progression. This study is therefore of high interest for urban planners and geologists who study sand dunes and require technical methods, based on machine learning and computer vision to allow them to collect large amount of data from satellite images to understand sand dunes progression and counter desertification problems. The use of cascading Haar feature provided a good accuracy, and the use of Support Vector Machines, along with the high resolution satellite images provided a good precision for the segmentation of barchan dunes contours, allowing the collection of morphological features which provide significant information on barchans sand dunes dynamics.
\end{abstract}

Keywords-Image segmentation; support vector machines; high resolution satellite images; remote sensing; sand dunes; desertification

\section{INTRODUCTION}

\section{A. The Study of Barchan Sand Dunes}

Barchan sand dunes are the fastest moving sand dunes, thus, they pose a problem to human settlements in desert arid and semi-arid regions around the world, as they could cover the farmlands, and cause the degradation of cultivated crops, damage houses, and trigger immigration. Also, moving sand dunes can cover intercity roads, which may create accidents, and block the road traffic for many hours, thus limiting the exchange of goods and restrict the economic development of arid and semi-arid regions. In fact, desertification affects around 250 million worldwide and 110 countries could be facing its consequences due to climate change [1]. Therefore, the study of sand dunes, and especially the fastest moving ones called barchans is important, and their large scale implies the use of advanced computer science and machine learning methods, in order to provide more relevant quantitative data, which can be used to assist specialists to understand better this phenomenon, and help decision makers to get an meaningful insight, and thus, contribute to find more efficient solutions to limit the impact of barchans dunes progression on human activities.

\section{B. Detection of Sand Dunes in Satellite Images}

The use of satellite imagery along with computer vision and digital image processing algorithms is essential to allow collecting data about sand dunes which are usually scattered on the surface of large deserts. Indeed, researchers have been using remote sensing imagery to study sand dunes and collect data more efficiently in such harsh environments: In the early attempts [2] used remote sensing to manually map sand dunes and develop their taxonomy, later, [3] started the detection of sand dune using their surfaces reflectance. In [4], pairs of ASTER satellite images were combined to extract barchans and collect their morphology measurements and study their dynamics. In [5], convex hulls were used to combine SRTM Digital Elevation Model and orthorectified Landsat TM, then $\mathrm{R}$-statistic index to estimate the degree of self-organization of dunes in Ar Rub' al Khali sand sea on the Arabian Peninsula. In [6], LiDAR was combined with spectral and spatial resolution of QuickBird satellite images, and optical stimulation luminescence was used to create an elevation model for Canadian barchans dunes. In [7], the mineral composition in Gran Desierto dune field in New Mexico, USA was mapped using ratio indexes on images from ASTER (Advanced Spaceborne Thermal Emission and Reflection Radiometer). In general, remote areas for which the access is sometimes impossible have benefited from using remote sensing along with computer vision and machine learning algorithms advancements. Indeed, availability of images from Landsat, MODIS, IKONOS, HIRISE, and MRO among others allowed not only to study dunes in difficult access areas such as the 
Namib Desert and Sahara Desert but also to discover the existence of barchans sand dunes in other planetary systems such as Mars: In [8] images from MRO (Mars Reconnaissance Orbiter) were used to study the seasonal modifications of Mars Northern Polar dunes thanks to high signal-to-noise ratio (SNR) in the dark dunes images. Dunes were also found in Venus: In [9], Cassini Radar images were used and contrasted black dunes to estimate their properties. We used in our previous work [10], the HIRISE (High-Resolution Imaging Science Experiment) satellite images to detect barchan dunes using MSER blobs. Barchans dunes tend to form group patterns [11], which complicates the detection of their position, as they usually overlap, which makes the use of more advanced segmentation methods necessary in order to distinguish dunes from their background.

\section{Image Segmentation and Machine Learning}

The spatial and spectral properties of satellite images can lead to good results for the detection and segmentation of sand dunes when the configuration of dunes is simple and the images are well contrasted. However, in many cases, the dunes are not in a stable system, and interact with vegetation and crust, therefore, the classical image detection approaches we introduced in the previous chapter face new challenges, especially in more complex dune fields, where the dunes are colliding. Hence, more advanced segmentation techniques were introduced in order to differentiate sand dunes from their surroundings. Researchers have tried to overcome these issues using different image segmentation approaches: In [12] pixel based segmentation was used to study Salinas-El Espartal dunes in the coast of Asturias, Spain. In [13], Maximum Likelihood and Minimum Distance Parallelepiped were demonstrated for classifying barchans. Sub-pixel analysis was extensively used to analyze dunes in Sinai Peninsula, Egypt [14]. In remote sensing, the use of methods based on objectoriented segmentation increased as the resolution and availability of satellite image improved, and the research switched progressively from the use of pixel-based methods to GEOBIA (Geospatial Object Based Image Analysis), allowing the use of more advanced image segmentation methods [15]. Image segmentation could be split into four mains classes: The region-based segmentation, edge-based segmentation and point-based segmentation, along with their combination [16]. Regions can be defined using homogeneity criteria based on a feature space, which is derived from the multi spectral satellite images, and thus have regions can encompass more information that segments, which in turn take advantage of spectral information to convey the mean, variance, median, minimum and maximum values per image bands, and thus, hold more information than single pixels. The Geospatial applications have been integrating segmentation methods developed for other domains such as pedestrian recognition for road safety [17], or for medical image analysis [18]. Hence, the models based on color and texture segmentation, and also relying on shape or motion [19] were used in satellite image processing. In fact, distances, neighborhood and topologies are essential for the object-based methods. Image segmentation has evolved with the availability of benchmark databases and the development of machine learning approaches, which required to be fed with features. Therefore segmentation methods were based on feature engineering, and many descriptors and detectors were developed such as Haralick features [19] which were invariant to quantization gray-levels number, and were calculated using GLCM (Gray-Level Co-occurrence Matrix), thus taking into account image texture. They were used for the discrimination of sand dunes in the Valdes peninsula [20]. The Histogram of Oriented Gradients (HOG) which became popular [21] and Local Binary Patterns (LBP) introduced in [22] served as descriptors and were also used in for the detection of sand dunes [23]. Later, other detectors and descriptors such as Scale-Invariant Feature Transform (SIFT) was used extensively in different computer vision applications [24] such as pedestrian detection, fingerprint recognition, however, at the extent of our knowledge, they were not used in sand dunes detection in satellite images. It is worth noting other important descriptors such as Speed-Up Robust Features (SURF) [25], played an important role. In fact, SIFT performs better than SURF for scale changes, but SURF has lower execution time than SIFT when it used integral images [26]. Fast Retina Keypoint (FREAK) which was introduced by [27] consists of series of Difference of Gaussians (DoG) which are composed over a retinal pattern, was also used in many applications in digital image processing and object recognition. and Binary Robust Independent Elementary Features (BRIEF) which is highly discriminative in the case of few bits [28], and There is also Binary Robust Invariant Scalable Keypoints (BRISK) [29] which has a lower computational cost, Oriented FAST and Rotated BRIEF (ORB) [30] which is rotation invariant and resistant to noise. Also, MSER (Maximally Stable Extremal Regions) detectors were efficient as their timesteps were chosen so as to achieve more stability against changing intensity in different scales, in addition to blur [31]. These descriptors were vectors which represented information, and thus, simple threshold were not very effective to classify complex objects and images. Indeed, the descriptor vectors were later followed by the use of machine learning algorithms, which had to be used generally in the paradigm of supervised learning, where the data was split into a learning set, a validation set and a test set [32]. The use of classification or regression techniques is generally based on LR (Logistic regression), SVM (Support Vector Machines), ANN Artificial Neural Networks, and their multiple variations such as CNN (Convolutional Neural Networks) which includes DL (Deep Learning). The classification of remote sensing images also made use of Markov Random Field (MRV) [33].

\section{Our Contribution}

As we showed in the previous paragraphs, there were many studies for quantifying sand dunes dynamics, but very few used computer vision and machine learning approaches, and no study in our knowledge went through the entire process from the detection of barchans dunes in satellite image, then image segmentation and finally the automatic measurement and comparison of features with allometry algorithms. Therefore, our contribution is an end to end process which starts from a raw satellite image, used supervised learning to detect dunes, segment their contours using SVM, and then collect they allometry with a dedicated algorithm. In the following chapters, we will provide the detailed steps of our approach, then, we will expose the experimental results and finally, we will give a glance at the upcoming direction of future works, and finally we will conclude. 


\section{PROPOSED WORK}

In the following flowchart Fig. 1, we depict the steps we used to achieve the final goal which is the automatic extraction of dunes measurements. Each of the three lanes represents one major step:

In the first lane, we detect the dunes locations in a satellite image: We start by splitting our high resolution satellite image randomly in two parts: The first part, which is about $70 \%$ of the total area, is used for training, and the remaining area, around $30 \%$, was put aside for testing and evaluating the learning. We then started by a simple image enhancement using Weiner filter, then, we manually annotated dunes in order to have a ground truth. Then, we used a cascade classifier based on Haar wavelets to learn the dune model parameters. The result was a trained model, which we evaluated on the test region, in order to get predicted detections of barchans dunes from the cascade classifier model. As we noticed that few dunes were detected more than once by the classifier, we added a processing step which is candidate's fusion, in order to combine overlapping bounding boxes. The result of this first step is thus a set of images containing the detected barchans sand dunes.

In the second lane, we started by using a machine learning approach and specifically supervised learning. We used the dunes annotation of bounding boxes from the first step, and annotated the contours in addition, to get a labelled learning set, and again, we left a test set for evaluation. We proceeded in the learning set and normalized the data, as dunes come in different sizes and shapes. We used shapes alignment, and later on dimensionality reduction in order to compress the information coming from the 2D shapes of dunes, thus extracting the most significant ones. The result was a shape model which describes a mean dune, and the variance which covered most of dune shapes. This result allowed us to have a check on the geometric information. From another hand, to recover the geometric position from the test images, we collected SIFT image descriptors in specific points. More accurately, we extracted SIFT vector descriptor around each annotated contour, and onto the contour itself, then labelled the SIFT points on the contour as positive, and those around the contour as negative.

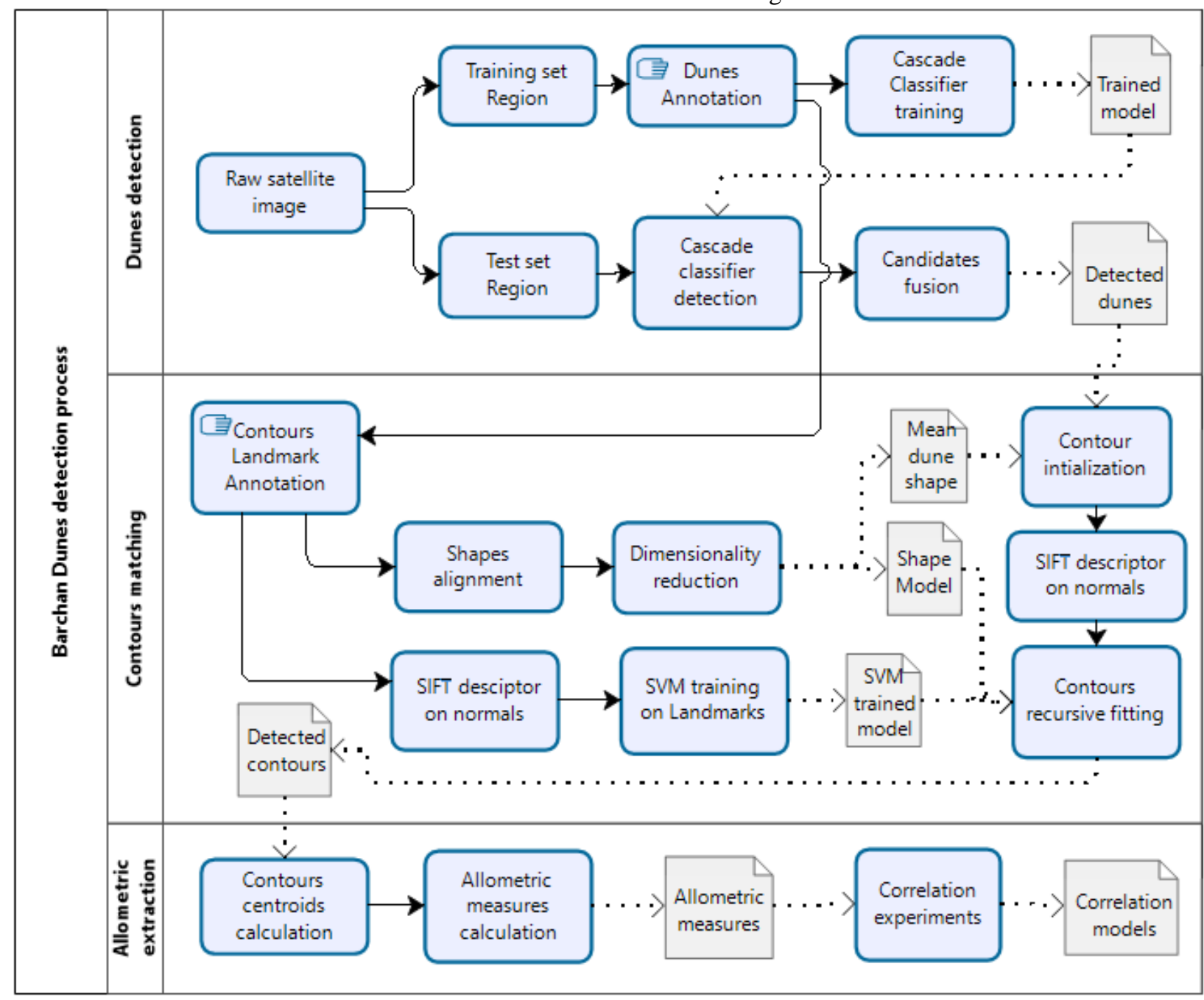

Fig. 1. Flowchart of the Process of Detection, Segmentation and Algorithmic Allometry of Barchans Sand Dunes. 
We then trained a Support Vector Machine to classify the SIFT descriptor points as belonging to a contour of a dune, or not if it belongs to the surroundings. The produced model was then used for predicting if the new SIFT points were belonging or not to the contour. Now that the SVM model, shape model, and the mean dune are ready, we proceed used the test images produced by the first step. For each of the test images, we started by plotting the mean dune that is our starting point, from which we searched to match the contours of the dune which was detected in the test image. For this purpose, we started by extracting SIFT features around the curve of the mean dune, along its normal, then we classified them using SVM, as belonging to the dune contour or not. Those who were positive were used to deform the mean dune, into a new shape, which was closer to the shape of the dune in the test image. Then, to check that the new shape was acceptable, we used the shape model produced with PCA to correct the dune shape, and keep it within the acceptable standard deviation of the dune model. Thus, if the shape deviated more than $3 \sigma$, it was set $3 \sigma$. This operation was done recursively in order to match the contours of the barchans dune, and we stopped after 10 iterations for each image as it was sufficient to converge toward the dune contour in the test image. The result for each dune image was its 3 contours called (Brink, Leeward and Windward.) contours.

In the third lane, we used the contours produced by the former step then calculated for each one, its centroid. These centroids along with the contours will be used by our algorithm which calculates the allometric measures of each dune. First, the line traversing the 3 centroids formed an axis, which we call the dune direction. The other metric automatically extracted are the width, found by drawing an orthogonal line to the direction passing by the crest, and intersecting with the outward contours of the dune. We calculated horns centroids, then horns width, as the sum of both horns distances. This measure was calculated as the distance between the parallel lines to the direction, passing respectively through a horn centroid and the outermost contour. Finally, we explored the collected data and extracted correlation models.

\section{EXPERIMENTAL RESULTS}

\section{A. Satellite Image Dataset}

We used an IKONOS high resolution satellite image from the satellite IKONOS, which is an Earth observation satellite used to collect imagery on multispectral (MS) and panchromatic (PAN) wavelengths. Its band resolutions consist of one panchromatic band with $0.8 \mathrm{~m}$ spatial resolution for 450-900 nm wavelengths, and 4 multispectral bands with $4 \mathrm{~m}$ spatial resolution with Near Infrared, Red, Green and Blue corresponding respectively to $757-853 \mathrm{~nm}, 632-698 \mathrm{~nm}, 506-$ $595 \mathrm{~nm}$ and $445-516 \mathrm{~nm}$ wavelengths. The location of the image was in the Sahara Desert, more precisely in the South of Morocco, and the limiting coordinates of the image correspond to $27^{\circ} 26^{\prime} 8.6621^{\prime \prime} \mathrm{N}, 13^{\circ} 08^{\prime} 5.2628^{\prime \prime} \mathrm{W}$ and $27^{\circ} 41^{\prime} 1.0350^{\prime \prime} \mathrm{N}$, $13^{\circ} 22^{\prime} 0720^{\prime} \mathrm{W}$. The date the image was taken on by the satellite was the 23/07/2003. The total area of the image corresponds approximately to $12 \mathrm{~km} 2$. The area contains hundreds of sand dunes, and we focused on a sub area where dunes were not colliding much. Our dataset consisted of 240 images with 120 sand dunes split into 80 sand dunes used as a learning set and 40 used as a test set, plus 120 images without sand dunes, containing mainly the flat desert surrounding the dunes.

\section{B. Metrics and Formula}

Barchan sand dunes are three dimensional objects, which appeared as 2D crescent like objects in satellite images. We therefore used an approach where we first scaled the multispectral images to the size of the panchromatic image using bicubic interpolation for $4 \times 4$ pixels using equation (1):

$p(x, y)=\sum_{i=0}^{3} \sum_{j=0}^{3} a_{i j} x^{i} y^{j}$

After enhancing the image using the median filter, we proceed and used a Haar classifier for the first step, which was based on selecting the best weak classifier with respect to the weighted error using equation (2):

$\varepsilon_{t}=\min _{f, p, \theta} \sum_{i} w_{i}\left|h\left(x_{i}, f, p, \theta\right)-y_{i}\right|$

The results were candidate dunes, surrounded by bounding boxes. They were combined when their overlapping was over $40 \%$. Then each selected bounding boxed was enlarged by $25 \%$ then all ROI (Regions of Interest) were normalized by resizing them to $128 \times 128$ pixels. In the second step, SIFT descriptors which are based on the difference of Gaussians in (3) were extracted in 10 equidistant points from the contours named landmarks, and 4 pixels along the normal of each landmark, which is a total of 50 SIFT point per dune image.

$G(x, y, k \sigma)-G(x, y, \sigma) \approx(k-1) \sigma^{2} \nabla^{2} G$

After the SIFT features were calculated, the dune shapes represented by their contours were aligned and SIFT descriptors were fed to an SVM, so as the descriptors falling on the contours were defined as positive examples, and the SURF descriptors falling on the normal surrounding the contours landmarks were defined as negative examples. The SVM maximized the separating hyper plan described in (4), where we used a linear Kernel.

$h(x)=\sum_{k=1}^{p} \alpha_{k}^{*} l_{k} K\left(x_{k}, x\right)+w_{0}$

Subsequently, for testing and evaluation, we used the dunes test examples, and calculated with Mahalanobis distance. Finally, in the third step, we used a set of normalized allometry features defined in Fig. 2, which were geometrical measures calculated with our algorithm. We produced for each dune, the vector $\mathrm{V}(\mathrm{d})$ in (5), with $\mathrm{w}, \mathrm{L}, \theta, \mathrm{h} 1, \mathrm{~h} 2$, ih, $\mathrm{S}$ corresponding respectively to Width, Length, Angle, Horn widths, inter-horns distance, and Surface area.

$V(d)=\left(w, L, \theta, h_{1}, h_{2}, i h, S\right)$

These measures are illustrated in Fig. 2 which shows, the centroids corresponding to different contours: $\mathrm{Cw}, \mathrm{Cb}, \mathrm{Cl}$ to windward, brink and leeward contours respectively, and Clb1, $\mathrm{Cw} 1$, to the extremities of leeward and brink, and the extremity of windward curved respectively. NS represents a hypothetical North-South axis. 


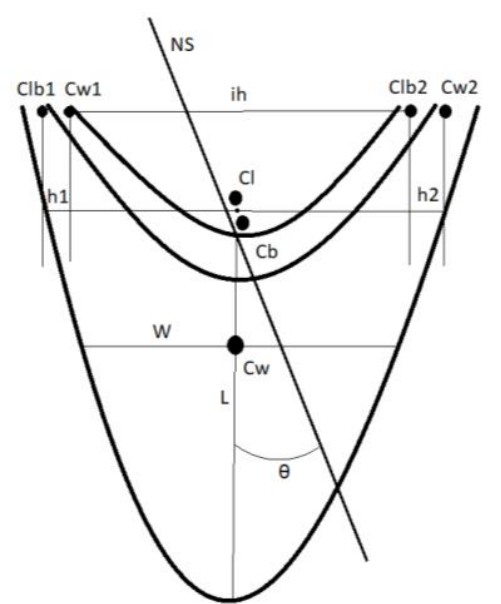

Fig. 2. Allometry Measures of Barchan sand Dunes Contours.

\section{Graphics and Comments}

We show in Table I the confusion matrix from which we calculated the accuracy of the dunes detection. When we used the fusion of dunes detection, the results returned for the dune detection increased from $65 \%$ to $77.5 \%$. This increase is explained by the fact that overlapping detection tends to create false positives, which were generally corresponding to parts of the dunes such as horns. Fusion was reducing the number of false negatives, thus improving the accuracy.

TABLE. I. CONFUSION MATRIX OF DUNES Detections

\begin{tabular}{|l|l|l|l|}
\hline \multirow{2}{*}{ Confusion matrix of dune detection } & Truth \\
\cline { 3 - 4 } & Positive & Negative \\
\hline \multirow{2}{*}{ Detection results before fusion } & Positive & 18 & 23 \\
\cline { 2 - 4 } & Negative & 5 & 34 \\
\hline \multirow{2}{*}{ Detection results after fusion } & Positive & 29 & 12 \\
\cline { 2 - 4 } & Negative & 6 & 33 \\
\hline
\end{tabular}

The goal of this study is to use machine learning and computer vision approaches in order to collect dunes measures (width, length, horns distances, ...) which may be used by other domain specialists. We therefore demonstrate in our own dataset a set of correlations found between these dunes features in Fig. 3.

The relationships found in Fig. 3 from top to bottom correspond respectively to the equations (6), (7) and (8).

$w=8.10^{-3}\left(h_{1}+h_{2}\right)^{2}+0.2\left(h_{1}+h_{2}\right)+7.1$

$S=10^{-3}\left(5.10^{-4} w^{2}+77.10^{-3} w-2.97\right)$

$w=19.89+0.85 i h$

These equations are the results which can be useful to domain experts and help decision makers to estimate the dunes risk and danger. Our contribution consists of the machine learning and computer vision approach organized in a process we illustrated in Fig. 4, describing each step. The original satellite image is enhanced in Fig. 4(a), then the use of a Haar classifier resulted in the detection of dunes indicated by the bounding boxes, the Fig. 4(c) is a zoomed in part of the result. For each bounding box, we normalized the image to $128 \times 128$ pixels in Fig. 4(d). Then, we extracted the SIFT descriptor features around the mean dunes, and along the normal, which we provided to a SVM (Support Vector Machine) in order to classify positive SIFT landmarks corresponding to the contours, and negative SIFT, not corresponding to the contour. Thus, by linking the SIFT positive points; we obtained a set of 3 curves represented in Fig. 4(f). Finally, we used an algorithm for measuring allometry features represented in Fig. 2, in order to normalize the data measures. These measures depicted by yellow segments in Fig. 4(f) are then stored in a vector (5). We then used the data which we found using our dunes detection and segmentation approach, to create a set of features vectors (5), and explored correlations.

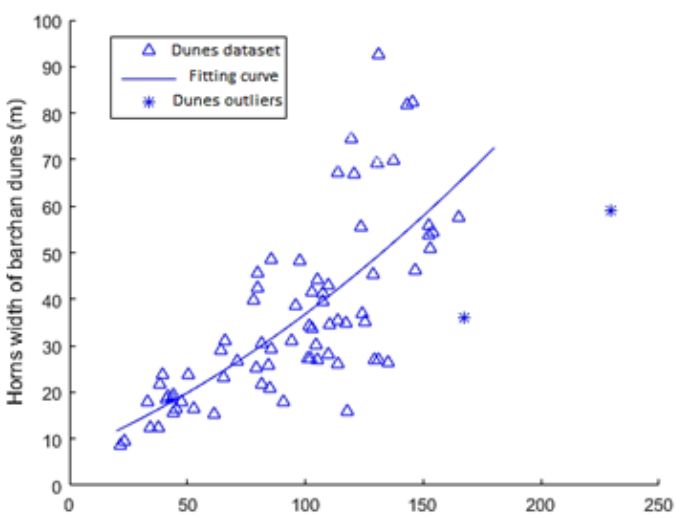

(a) Width of Barchan Dunes (m).

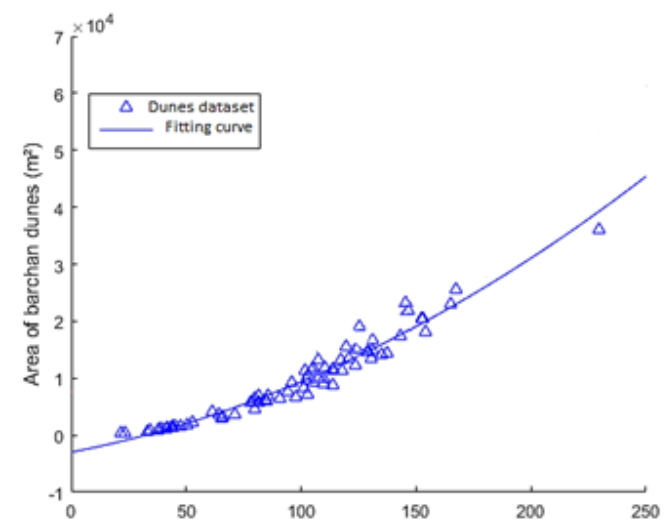

(b) Width of Barchan Dunes (m).

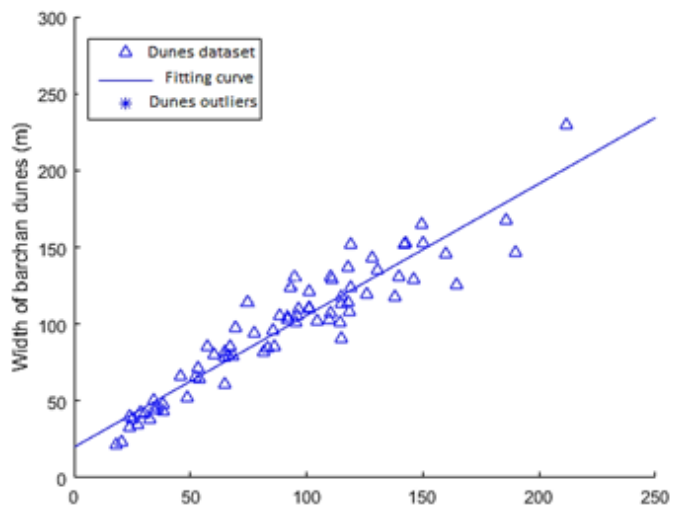

(c) Inter-Horns Distance of Barchan Dunes (m).

Fig. 3. Dunes data feature correlations examples: (a): Width as a function of horns width: $\mathrm{f}(\mathrm{w})=\mathrm{h} 1+\mathrm{h} 2(\mathrm{~b})$ : Area as a function of width: $\mathrm{f}(\mathrm{w})=\mathrm{S}$ (c): Width as a function of inter-horns: $\mathrm{f}(\mathrm{ih})=\mathrm{w}$ 
In Fig. 5, we exposed the results we obtained after using our computer vision approach, which allowed us to determine dunes measures, after detecting and segmenting dunes from high resolution satellite images using SVM. We showed in Fig. 5(a), one of the most interesting results we obtained: After collecting data about the surface size of dunes as a function of their angle, we found a normal like distribution where the largest dunes were centered on $\theta=22.5$ degrees (or NNE). Moreover, the histogram in Fig. 5(c) produced by data collected using our method also showed that most of the dunes are centered on the same angle $\theta$. As it was a specific numbers, we decided to further search for an external data source on to confirm or infirm the results obtained by our method of data collection. Thus, we found that the wind rose in Fig. 5(b) which provided us with the wind direction in the region from which our dataset originated in the satellite image, showed the wind blowing mainly from 22.5 degrees (or NNE). Therefore, it confirmed that our approach based on machine learning and computer vision process to automated process of data collection from satellite images could very accurately measure barchans dunes allometry features, and obtain results, which were coherent with the field.

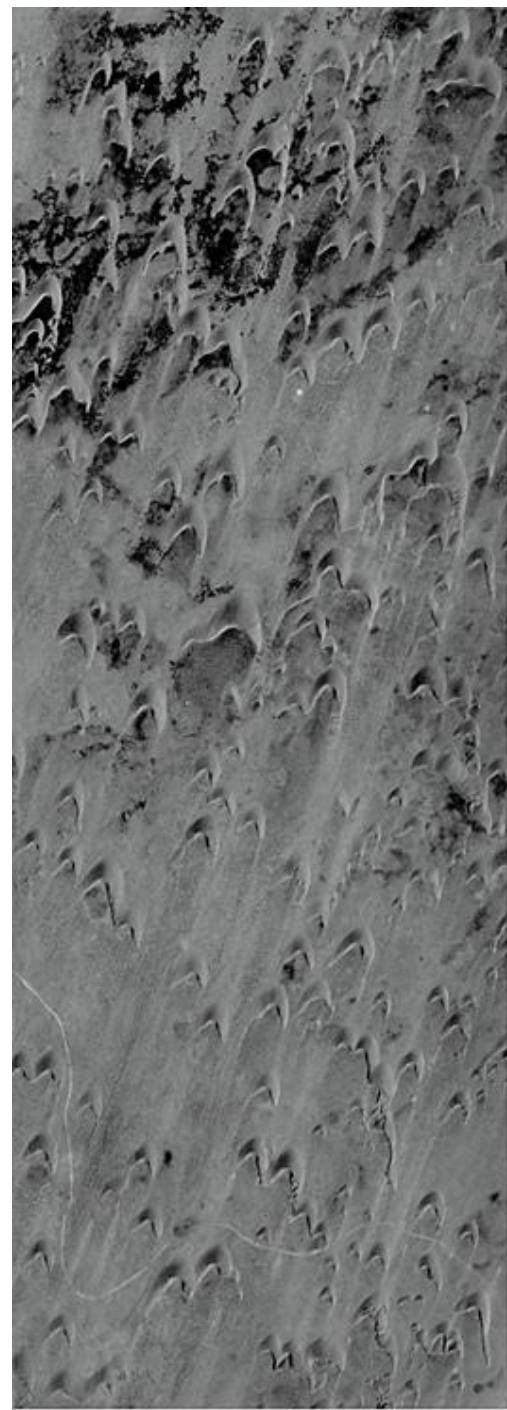

a

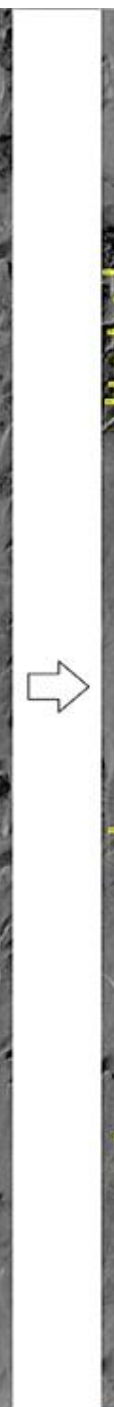

b

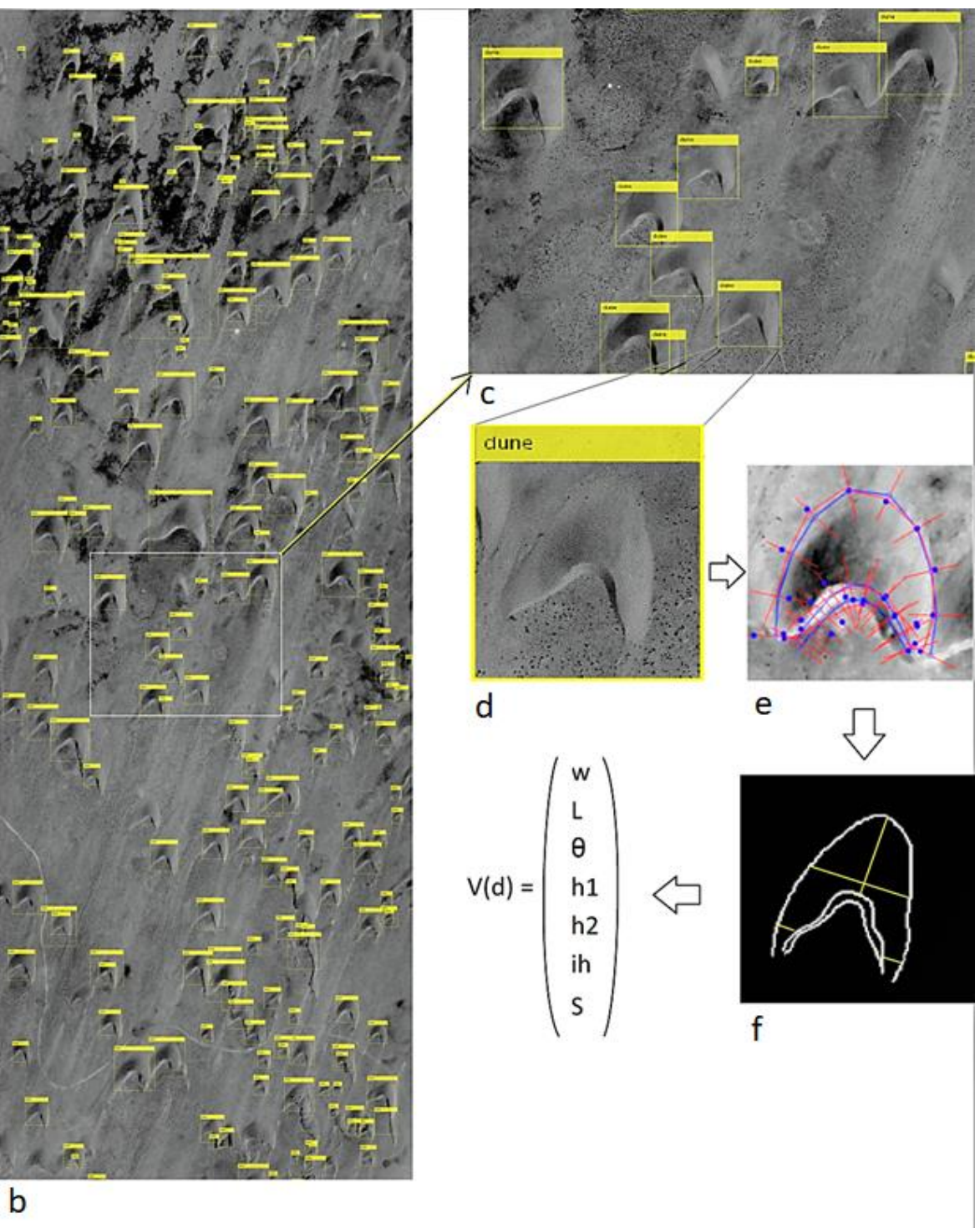

Fig. 4. Image Detection and Contours Segmentation Process for Collecting Dunes Measurements (a): The Satellite Image after Image Enhancement (b): The Satellite Image after Dunes Detection using Haar Wavelets (c): A Zoomed in Portion of the Satellite Image in 2 (d): The Dunes after ROI Normalization to 128x128 Pixels (e): Red Lines are Normals Along which SIFT Descriptors were Calculated. Blue Dots Represent the SIFT Classified as Positive using SVM, Forming Blue Contours (f): Resulting Dune Contours are in White, Dunes Features Measured by our Algorithm are Yellow Segments, Final Result is a Vector (Width , Length, Widths of Horns). 


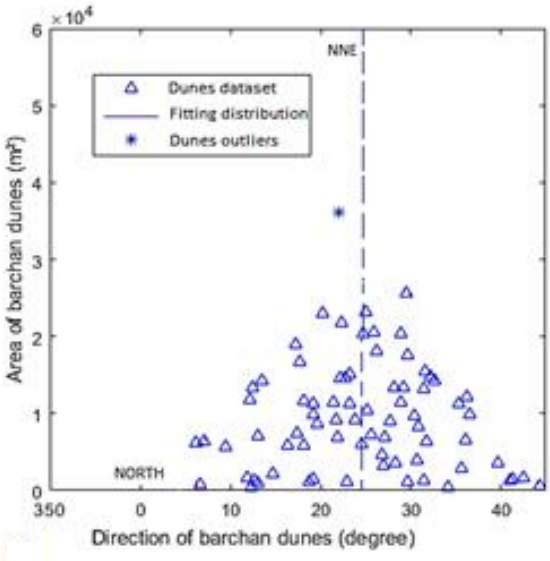

(a)

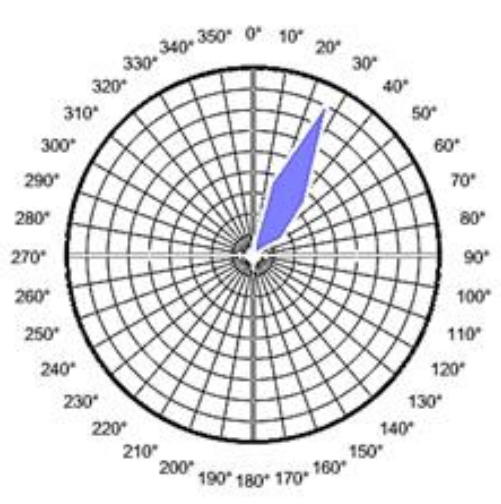

(b)

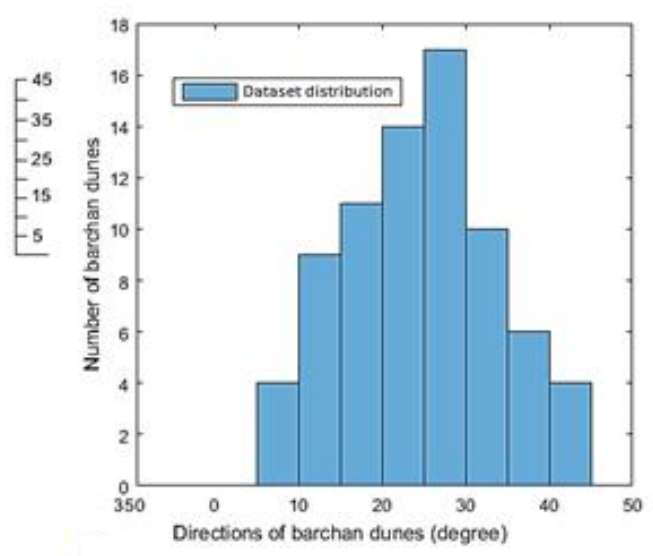

(c)

Fig. 5. Measures of Barchan Sand Dunes Contours (a): Surface Area of Dune as a Function of its Angle (b): The Wind Rose of the Region of our Dataset (c):The Histogram of Dunes Angles.

\section{CONCLUSION AND FUTURE WORK}

In this paper, we used a computer vision and machine learning approach for the detection, segmentation of sand dunes in high resolution satellite images. Our contribution is an end to end process which starts from the raw high resolution satellite image, which we enhanced, then using a Haar classfier we found dunes candidates, then for each validated candidate, we segmented its contours using SIFT features and an SVM. Moreover, we used an algorithm to measure the allometry of dunes, and finally explored the correlations. Our approach obtained a good accuracy of $77.5 \%$, which we could improve by taking into account dunes which are colliding in future works. Our approach allowed automating the collection of an important amount of data from a high resolution satellite image. The data results were confirmed by field measurement of wind rose. Finally, we found data relationships that we formalized with equations, which desertification experts can rely on to assess the risk of barchans sand dunes and take anticipated actions to protect the populations. Indeed, as smaller dunes tend to move faster, the collected data could identify the dunes having smaller width which may present higher risk.

The overall approach returned satisfactory accuracy results. However, we observed some limitations to our method due to cases of dunes having odd shapes. These deformed dunes are often the result of collision between dunes advancing at different speeds. In future work, we would use more advanced machine learning methods such as Deep Learning for the detection and segmentation of sand dunes in order to improve recall. Also, the use of several high resolution satellite images would allow using a multi temporal analysis of sand dunes.

\section{REFERENCES}

[1] UNCCD: United Nations Convention to Combat Desertification in those countries experiencing serious drought and/or desertification, particularly in Africa. UNCCD explanatory leaflet, UNCCD Secretariat, Bonn (2008).

[2] McKee, E.D., 1979. Introduction to a study of global sand seas. In: McKee, E.D. (Ed.), A Study of Global Sand Seas: United States Geological Survey, Professional Paper, 1052, pp. 3-19.
J. Clerk Maxwell, A Treatise on Electricity and Magnetism, 3rd ed., vol. 2. Oxford: Clarendon, 1892, pp.68-73.

[3] Blumberg, D.G., 1998. Remote sensing of desert dune forms by polarimetric Synthetic Aperture Radar (SAR). Remote Sensing of Environment 65, 204-216.

[4] Vermeesch, P., Drake, N., 2008. Remotely sensed dune celerity and sand flux measurements of the world's fastest barchans (Bodéle, Chad). Geophysical Research Letters 35, L24404.

[5] Bishop, M.A., 2010. Comparative nearest neighbor analysis of megabarchanoid dunes, Ar Rub al Khali sand sea: the application of geographical indices to the understanding of dune field selforganization, maturity and environmental change. Geomorphology 120, 186-194.

[6] Wolfe, S.A., Hugenholtz, C.H., 2009. Barchan dunes stabilized under recent climate warming on the northern Great Plains. Geology 37, 10391042.

[7] Scheidt, S., Ramsey, M., Lancaster, N., 2010. Determining soil moisture and sediment availability at White Sands Dune field, New Mexico, from apparent thermal inertia data. Journal of Geophysical Research 115, F02019.

[8] Hansen, C.J., Bourke,M.C., Bridges, N.T., Byrne, S., Colon, C., Diniega, S., Dundas, C., Herkenhoff,K., McEwen, A., Mellon, M., Portyankina, G., Thomas, N., 2011. Seasonal erosion and restoration of Mars' Northern Polar Dunes. Science 331, 575- 578.

[9] Bourke, M.C., Edgett, K.S., Cantor, B.A., 2008. Recent aeolian dune change on Mars. Geomorphology 94, 247-255.

[10] Azzaoui, M. A., Adnani, M., El Belrhiti, H., Chaouki, I. E., and Masmoudi, L.: Detection Of Crescent Sand Dunes Contours In Satellite Images Using An Active Shape Model With A Cascade Classifier, Int. Arch. Photogramm. Remote Sens. Spatial Inf. Sci., XLII-4/W12, 17-24, https://doi.org/10.5194/isprs-archives-XLII-4-W12-17-2019, 2019.

[11] Hugenholtz CH, Levin N, Barchyn TE, Baddock M, 2012. Remote sensing and spatial analysis of aeolian sand dunes: a review and outlook. Earth-Science Reviews 111: 319-334.

[12] Flor-Blanco, Germán \& Flor, Germán \& Pando, Luis. 2012. Evolution of the Salinas-El Espartal and Xagó beach/dune systems in northwestern Spain over recent decades: Evidence for responses to natural processes and anthropogenic interventions. Geo-Marine Letters. 33. 10.1007/s00367-012-0301-3.

[13] John A. Richards, Xiuping Jia. 2006. Remote Sensing Digital Image Analysis. 10.1007/3-540-29711-1.

[14] ElSayed Hermas, Sebastien Leprince, Islam Abou El-Magd, Retrieving sand dune movements using sub-pixel correlation of multi-temporal optical remote sensing imagery, northwest Sinai Peninsula, Egypt, Remote Sensing of Environment,Volume 121, 2012, Pages 51-60, ISSN 0034-4257. DOI: 10.1016/j.rse.2012.01.002. 
[15] T. Blaschke, Object based image analysis for remote sensing, ISPRS Journal of Photogrammetry and Remote Sensing, Volume 65, Issue 1, 2010, Pages 2-16, ISSN 0924-2716, DOI: 10.1016/j.isprsjprs .2009.06.004.

[16] Schiewe, Jochen. (2012). Segmentation of high-resolution remotely sensed data - Concepts, applications and problems. International Archives of Photogrammetry and Remote Sensing. 34.

[17] Benenson, R., Omran, M., Hosang, J., Schiele, B.: Ten years of pedestrian detection, what have we learned? In: Agapito, L., Bronstein, M.M., Rother, C. (eds.) ECCV 2014 Workshops. LNCS, vol. 8926, pp. 613-627. Springer, Heidelberg (2015).

[18] D. Bouget, R. Benenson, M. Omran, L. Riffaud, B. Schiele and P. Jannin, "Detecting Surgical Tools by Modelling Local Appearance and Global Shape," in IEEE Transactions on Medical Imaging, vol. 34, no. 12, pp. 2603-2617, Dec. 2015. doi: 10.1109/TMI.2015.2450831.

[19] Haralick, R.M.; Shanmugam, K.; Dinstein, I. Textural Features for Image Classification. IEEE Trans. Syst.1973, SMC-3, 610-621.

[20] P. D. Blanco , G. I. Metternicht, H. F. del Valle, W. Sione. 2007. Assessment of TERRA-ASTER and RADARSAT imagery for discrimination of dunes in the Valdes peninsula: an object oriented approach Revista de Teledetección. ISSN: 1133-0953. 2007. 28: 87-96.

[21] N. Dalal and B. Triggs. Histograms of oriented gradients for human detection. In CVPR, 2005.

[22] T. Ojala, M. Pietikäinen, and D. Harwood (1994), "Performance evaluation of texture measures with classification based on Kullback discrimination of distributions", Proceedings of the 12th IAPR International Conference on Pattern Recognition (ICPR 1994), vol. 1, pp. $582-585$.

[23] M. A. Azzaoui, M. Adnani, H. El Belrhiti, I. E. Chaouki, L. Masmoudi. 2018. Detection Of Crescent Sand Dunes Contours In Satellite Images Using An Active Shape Model With A Cascade Classifier. The International Archives of the Photogrammetry, Remote Sensing and Spatial Information Sciences, Volume XLII-4/W12, 2019.
[24] Lowe, David G. (1999). "Object recognition from local scale-invariant features" (PDF). Proceedings of the International Conference on Computer Vision. 2. pp. 1150-1157. doi:10.1109/ICCV.1999.790410.

[25] Herbert Bay, Andreas Ess, Tinne Tuytelaars, and Luc Van Gool. 2008. Speeded-Up Robust Features (SURF). Comput. Vis. Image Underst. 110, 3 (June 2008), 346-359. DOI=http://dx.doi.org/10.1016/ j.cviu.2007.09.014.

[26] Darshana Mistry, Asim Banerjee. Comparison of Feature Detection and Matching Approaches: SIFT and SURF. GRD Journals- Global Research and Development Journal for Engineering | Volume 2 | Issue 4 | March 2017 ISSN: 2455-5703.

[27] Alahi, Alexandre \& Ortiz, Raphael \& Vandergheynst, Pierre. (2012). FREAK: Fast retina keypoint. Proceedings of the IEEE Computer Society Conference on Computer Vision and Pattern Recognition. 510517. 10.1109/CVPR.2012.6247715.

[28] Michael Calonder, Vincent Lepetit, Christoph Strecha, and Pascal Fua, "BRIEF: Binary Robust Independent Elementary Features", 11th European Conference on Computer Vision (ECCV), Heraklion, Crete. LNCS Springer, September 2010.

[29] Stefan Leutenegger, Margarita Chli and Roland Siegwart: BRISK: Binary Robust Invariant Scalable Keypoints. ICCV 2011: 2548-2555.

[30] Ethan Rublee, Vincent Rabaud, Kurt Konolige, Gary R. Bradski: ORB: An efficient alternative to SIFT or SURF. ICCV 2011: 2564-2571.

[31] Per-Erik Forssén. Maximally stable colour regions for recognition and matching. In Computer Vision and Pattern Recognition, 2007. CVPR'07. IEEE Conference on, pages 1-8. IEEE, 2007.

[32] Abu-Mostafa, Y. S., Magdon-Ismail, M., \& Lin, H. (2012). Learning from data: a short course. [United States]: AMLBook.com.

[33] Li, C.; Wang, J.; Wang, L.; Hu, L.; Gong, P. Comparison of Classification Algorithms and Training Sample Sizes in Urban Land Classification with Landsat Thematic Mapper Imagery. Remote Sens. 2014, 6, 964-983. 